\title{
Documenting the elimination of measles, rubella and congenital rubella syndrome in Ontario: 2009-12
}

\author{
Lim $\mathrm{GH}^{1 *}$, Deeks $\mathrm{SL}^{1,2}$, Fediurek $\mathrm{J}^{1}$, Gubbay $\mathrm{J}^{1,3,4}$ and Crowcroft $\mathrm{NS}^{1,2,4}$ \\ ${ }^{1}$ Public Health Ontario, Toronto, Ontario \\ 2 Dalla Lana School of Public Health, University of Toronto, Toronto, Ontario \\ ${ }^{3}$ Department of Paediatrics, University of Toronto, Toronto, Ontario \\ ${ }^{4}$ Department of Laboratory Medicine and Pathobiology, University of Toronto, Toronto, Ontario \\ * Corresponding author: Gillian.lim@oahpp.ca
}

\section{Abstract}

Background: Under the guidance of the Pan American Health Organization (PAHO), countries of the Americas are currently documenting the elimination of measles, rubella, and congenital rubella syndrome.

Objective: This paper describes Ontario's progress in documenting the elimination of these conditions between 2009 and 2012.

Methods: All possible case classifications, including those that did not meet surveillance case definitions, were extracted from the provincial reportable disease database, the integrated Public Health Information System (iPHIS). Data were analyzed against select criteria specified by the PAHO, specifically with respect to epidemiology, vaccination coverage, and the quality of the surveillance system.

Results: There were no known endemic cases of measles, rubella or congenital rubella syndrome reported in Ontario during the study period. Cases were predominantly the result of importation, as these diseases remain endemic in many countries. A total of 27 confirmed cases of measles were reported over the four-year period, most of which could be verified as being directly or indirectly linked with travel outside Canada. In addition, five confirmed cases of rubella and one case of imported congenital rubella syndrome were identified. A review of immunization coverage and surveillance data quality identified gaps. The combined annual rates of suspected measles and rubella cases (between 0.7 and 1.1 cases per 100,000 population) and suspected congenital rubella syndrome cases ( 0.21 to 0.49 cases per 10,000 live births) were below PAHO's thresholds. Also, the frequent absence of immunization and travel histories within iPHIS was of concern ( $77.3 \%$ and $44.6 \%$ respectively).

Conclusion: These results support Ontario's sustained elimination status. However, in order to satisfactorily meet PAHO's requirements for documenting the elimination of these diseases, continued vigilance is required. Efforts are currently under way within Ontario to improve reporting.

\section{Introduction}

The Region of the Americas adopted the goal of eliminating measles by 2000 , and rubella and congenital rubella syndrome by 2010 . The interruption of endemic measles virus was achieved in the Americas in 2002 while the last confirmed endemic rubella case was reported in Argentina in February 2009 (1). The last endemic measles and rubella cases were reported in Canada in 1997 and 2005 respectively (2).

In order to achieve measles elimination two doses of measles-containing vaccine are required, whereas a single dose of rubella-containing vaccine is required for elimination of rubella and congenital rubella syndrome $(3,4)$. In Canada, measles and rubella vaccines are available only in combination with mumps, or with mumps and varicella vaccine (MMR and MMRV respectively). In Ontario, a single dose of the combined MMR vaccine was implemented in 1975 followed by a two-dose program in 1996. The first dose of the MMR vaccine is routinely given at 12 months of age, while the timing of the second dose has varied between 18 months and 4-6 years of age (5). Under the guidance of the Pan American Health Organization (PAHO), countries of the Americas are 
currently documenting the elimination of measles, rubella, and congenital rubella syndrome. PAHO and the World Health Organization (WHO) developed a Plan of Action (6) for the documentation and verification of measles, rubella, and congenital rubella syndrome elimination (hereafter referred to as "the Plan"). The Plan provides guidance regarding the necessary evidence to verify that transmission of endemic measles and rubella viruses has been interrupted, and specifies several criteria and indicators that must be satisfied. The objective of this study was to evaluate Ontario's progress in documenting elimination against a subset of the criteria specified by PAHO's Plan of Action, using data that were readily available for analysis. The scope of the investigation was thus limited to an assessment of the epidemiology, vaccination coverage, and quality of surveillance for these diseases. We analyzed data between 2009 and 2012 to satisfy PAHO's criteria that the absence of endemic transmission must be demonstrated for at least three years.

\section{Methods}

To assess the epidemiology of measles, rubella, and congenital rubella syndrome in Ontario, we extracted all reports (regardless of case classification) between 2009 and 2012 from the provincial reportable disease database, the integrated Public Health Information System (iPHIS), as of June 3, 2013. Cases were classified as confirmed using provincial case definitions specified in Appendix B of the Ontario Infectious Diseases Protocol (7). Immunization information and travel histories were based on a review of several fields in iPHIS, including free text fields. Descriptive analyses presenting temporal, geographic, and demographic trends were limited to confirmed cases. An imported case was defined as a case who had traveled outside Canada 7-21 and 14-21 days before symptom onset for measles and rubella respectively. These definitions were modified from those in the Plan to reference travel outside Canada rather than the Americas and to be consistent with the incubation periods specified in the Infectious Diseases Protocol (7). An import-related case was one that resulted from transmission by an imported case (i.e. epidemiologically linked). If a chain of transmission spanned 12 months or longer, cases would be considered endemic.

Within the context of elimination, we considered a single confirmed case as an outbreak, even in the absence of subsequent disease transmission. For measles, we applied the methodology described by De Serres et al. (8) to estimate the effective reproductive number $\left(R_{e}\right)$; outbreaks with no subsequent transmission (i.e. $N=1$ ) were also included in this analysis. $R_{e}$ was derived using maximum likelihood estimation; $95 \%$ confidence intervals were also estimated.

For the purpose of this analysis Canadian guidelines were applied, requiring immunization coverage with two doses of measles vaccine and a single dose of rubella vaccine, to satisfy PAHO criteria using 2011-12 coverage data among 7- and 17-year-olds from the provincial Immunization Record Information System (IRIS); the number of doses required for adequate coverage is not explicitly stated in the Plan.

All suspected and confirmed case classifications of measles, rubella, and congenital rubella syndrome were analyzed when assessing the quality of surveillance. Table 1 lists the specific indicators and minimum thresholds required to satisfy PAHO criteria. Adequate investigation was determined on the basis of the data elements that were available in iPHIS; genotype information on outbreaks was sourced from the Public Health Ontario Laboratories. 
Table 1: Documenting elimination in Ontario: components of PAHO's Plan of Action that were within the scope of this study

\begin{tabular}{|c|c|}
\hline Component & Indicators and suggested analyses \\
\hline $\begin{array}{l}\text { Epidemiology of measles, } \\
\text { rubella and congenital } \\
\text { rubella syndrome (CRS) }\end{array}$ & $\begin{array}{l}\text { Verify the interruption of endemic measles, rubella, and CRS cases for a period of at } \\
\text { least } 3 \text { years from the last known endemic case, through an examination of } \\
\text { - Morbidity rates } \\
\text { - Temporal and spatial characteristics } \\
\text { - Seasonality } \\
\text { - Demographic characteristics } \\
\text { - Outbreaks }\end{array}$ \\
\hline $\begin{array}{l}\text { Measles and rubella } \\
\text { vaccinated population } \\
\text { cohorts }\end{array}$ & $\begin{array}{l}\text { Population cohort aged less than } 40 \text { years with ideally at least 95\% } \\
\text { coverage. }\end{array}$ \\
\hline $\begin{array}{l}\text { Quality of measles, rubella } \\
\text { and CRS surveillance }\end{array}$ & $\begin{array}{l}\text { Reporting rate } \\
\text { - Annual rate of suspected measles and rubella cases >= } 2 \text { per } 100,000 \\
\text { population } \\
\text { - Annual rate of suspected CRS cases >= } 1 \text { per } 10,000 \text { live births } \\
\text { Adequate investigation } \\
\text { - } \% \text { suspected measles and rubella cases with the following } 11 \text { data points } \\
\text { completed: name and/or identifier, place of residence, sex, age or date of } \\
\text { birth, date of reporting, date of investigation, date of rash onset, date of } \\
\text { specimen collection, presence of fever, date of prior MR vaccination, and } \\
\text { travel history } \\
\text { \% suspected CRS cases with the following eight data points completed: } \\
\text { name and/or identifier, place of residence, sex, date of birth, date of } \\
\text { reporting, date of investigation, date of specimen collection, and vaccination } \\
\text { history of mother; also clinical examinations for deafness, blindness, and } \\
\text { congenital cardiopathy >=80\% } \\
\text { Viral detection } \\
\text { \% measles and rubella outbreaks with genotype information available from at } \\
\text { least one viral specimen } \\
\text { \% confirmed congenital rubella syndrome cases with adequate specimen } \\
\text { analyzed for virus detection/isolation >= } 80 \%\end{array}$ \\
\hline
\end{tabular}

We compiled and analyzed all data in SAS version 9.2 and Microsoft Excel 2010. Incidence rates of measles and rubella were calculated using demographic data from Statistics Canada, accessed through IntelliHealth Ontario. Incidence rates of congenital rubella syndrome were determined using live births data from Statistics Canada. 


\section{Results}

\section{Measles epidemiology}

Between 2009 and 2012, 27 confirmed and nine probable cases of measles were reported in Ontario. In addition, 316 cases were investigated but did not meet the case definition. Among confirmed cases, the annual incidence rate between 2009 and 2012 was 0.54 (2009), 0.68 (2010), 0.60 (2011), and 0.22 (2012) cases per 1,000,000 population. Two thirds of the cases were female $(18 / 27,66.7 \%)$. The annualized incidence rate was 0.34 and 0.67 cases per 1,000,000 among males and females respectively. The median age of cases was 13.6 years, ranging between six months and 59 years. The highest annualized age-specific incidence rate occurred among infants $<1$ year of age $(7.0$ cases per $1,000,000)$. Immunization status could only be assessed for 19 of the 27 cases (70.4\%). Of these, $13(68.4 \%)$ were unimmunized, three (15.8\%) had had one dose, and three (15.8\%) had received two doses of measles-containing vaccine (up-to-date). Three of the unimmunized cases had a history of travel, and were under one year of age and therefore too young to have received routine MMR vaccine.

During the period of investigation, three outbreaks associated with at least one chain of transmission stemming from importation resulted in a total of 17 cases of measles. An additional 10 cases were reported that did not result in further transmission. Analysis of data from these 13 outbreaks revealed an estimated $R_{e}$ of $0.52(95 \%$ confidence interval $0.29,0.83$ ).

Importation status could be determined for all but two cases (25/27 or $92.6 \%)$ (Figure 1). If importation status had been defined solely on the basis of travel outside the Americas, as per the original PAHO definition, only $66.7 \%$ of cases (18/27) would have been classified as imported or import-related.

Figure 1: Confirmed Ontario measles cases by onset date and importation status, 2009-12 ( $\mathrm{N}=27$ )

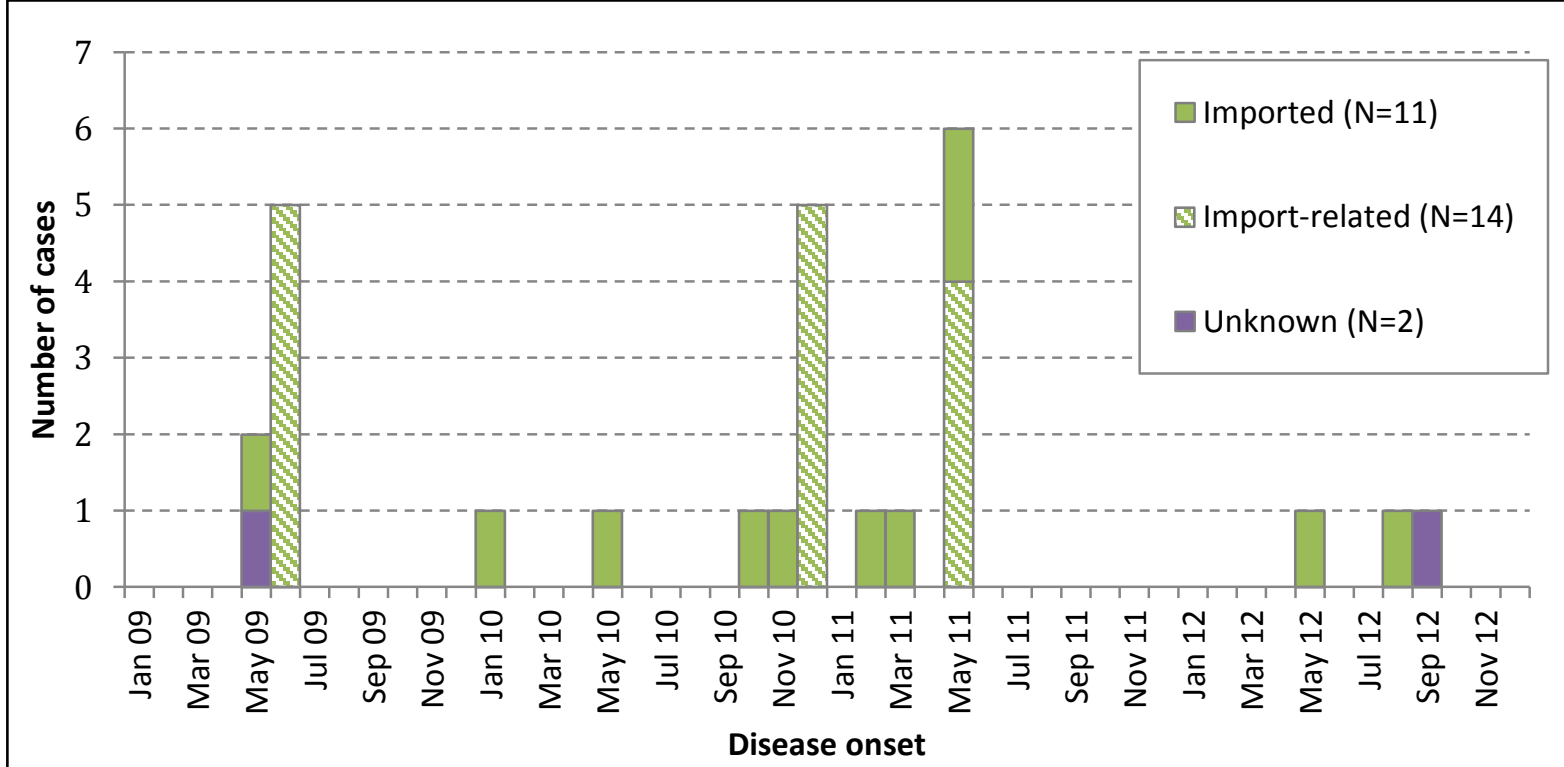


Two distinct episodes of importation were associated each with the United Kingdom, France, and Pakistan (Table 2).

Table 2: Travel histories and genotype information for confirmed cases of measles and rubella in Ontario that were found to be imported, 2009-12 ( $N=15)$

\begin{tabular}{|c|c|c|c|c|}
\hline Imported case ID & $\begin{array}{l}\text { Episode } \\
\text { month }\end{array}$ & Country of travel & Genotype & Number of subsequent cases \\
\hline \multicolumn{5}{|l|}{ MEASLES } \\
\hline 1 & May 2009 & $\begin{array}{l}\text { Exposure to measles case from } \\
\text { United Kingdom during travel in } \\
\text { United States }\end{array}$ & D4 & 5 \\
\hline 2 & Jan 2010 & Pakistan & & \\
\hline 3 & May 2010 & Sri Lanka & & \\
\hline 4 & Oct 2010 & France & & \\
\hline 5 & Nov 2010 & Philippines & D9 & 5 \\
\hline 6 & Feb 2011 & United Kingdom & D9 & \\
\hline 7 & Mar 2011 & India & & \\
\hline 8 & May 2011 & United States & & \\
\hline 9 & May 2011 & France & & 4 \\
\hline 10 & May 2012 & Pakistan & B3 & \\
\hline 11 & Aug 2012 & Afghanistan & & \\
\hline \multicolumn{5}{|l|}{ RUBELLA } \\
\hline 1 & Jan 2009 & Sri Lanka and India & & \\
\hline 2 & Apr 2009 & India & & \\
\hline 3 & Apr 2010 & Bangladesh & & \\
\hline 4 & Jan 2012 & Russia and Belarus & & \\
\hline
\end{tabular}

Genotype information was reported for only seven cases; of these, genotype D9 was identified in four cases (57.1\%), D4 in two cases (28.6\%) and genotype B3 in one case (14.3\%). Overall, the genotype was known for only four of the 13 outbreaks $(30.8 \%)$.

\section{Rubella epidemiology}

Between 2009 and 2012, five confirmed cases and one probable case of rubella were reported in Ontario. In addition, 139 cases were investigated but did not meet the case definition. Among confirmed cases, the annual incidence rate between 2009 and 2012 was 0.23 (2009), 0.08 (2010), 0.00 (2011) and 0.07 (2012) cases per $1,000,000$. No secondary transmission occurred. There was an equal distribution of male and female rubella cases, excluding one case for which the sex was unknown. The median age of cases was 28.3 years and ranged between 22 and 54 years. 
Figure 2 presents the distribution of cases by month of disease onset and importation status. Travel to Russia and Belarus, India, Sri Lanka, and Bangladesh were implicated as the source of exposure for the imported cases; genotype information was not reported for any of the cases in iPHIS (Table 2). For two cases, the reported country of birth was consistent with the country of travel (India and Bangladesh). Immunization status could be determined for only one case $(20 \%)$, who was unimmunized.

Figure 2: Confirmed Ontario rubella cases by onset date and importation status, 2009-12 (N=5)

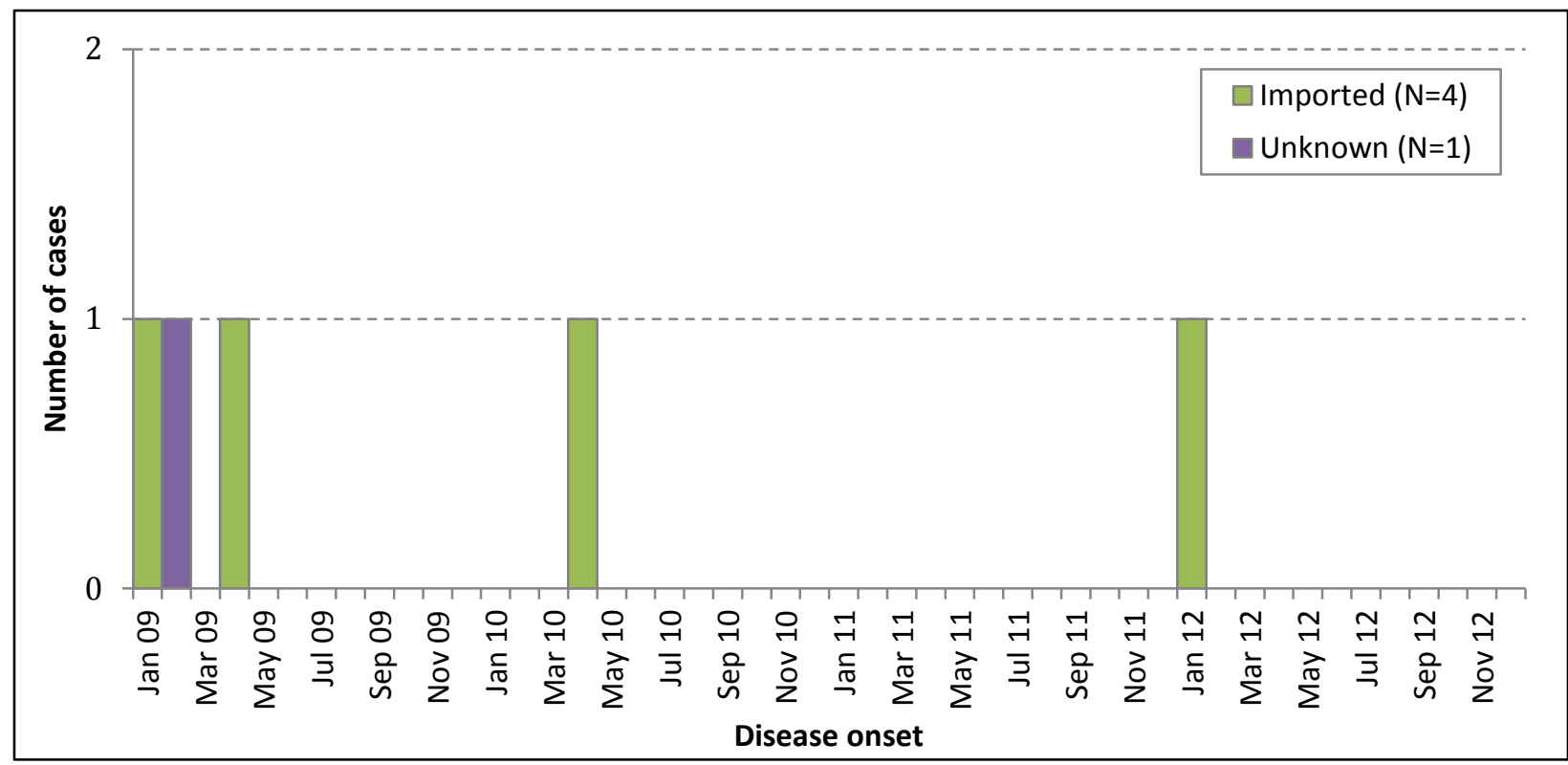

\section{Congenital rubella syndrome epidemiology}

Only one confirmed case of congenital rubella syndrome was reported in iPHIS during the period under surveillance, and this occurred in 2009. It was found to be an imported case, since the mother travelled outside of Canada during her pregnancy. A further 16 cases were investigated but did not meet the case definition. This equates to an incidence of 0.07 cases of congenital rubella syndrome per 10,000 live births in 2009 and an annualized incidence of 0.02 congenital rubella syndrome cases per 10,000 live births over the entire study period.

\section{Vaccine coverage}

Coverage estimates from the 2011-12 school year indicate that between the ages of seven and 17 years, twodose measles-containing vaccine coverage ranged between $89.1 \%$ among students seven years of age and $95.0 \%$ among students 16 years of age. Single-dose rubella vaccine coverage ranged between $95.1 \%$ among seven-year-olds and $96.9 \%$ among both 15- and 16-year old students. Single-dose measles coverage was similar to that of rubella.

\section{Quality of surveillance}

The combined annual rate of suspected measles and rubella cases ranged between 0.7 and 1.1 cases per 100,000 ; the annual reporting rate of suspected congenital rubella syndrome cases ranged between 0.21 and 0.49 cases per 10,000 live births. Genotype information was available for only four of $18(22.2 \%)$ measles and rubella outbreaks.

Only $2.4 \%(\mathrm{~N}=12)$ of suspected measles and rubella cases had all the required 11 data elements recorded within iPHIS. None of the suspected congenital rubella syndrome cases could be assessed, as some of the required data elements were not captured in iPHIS. Table 3 identifies the highest proportion of missing or incompletely 
recorded data elements, which included travel history (77.3\%), the date on which a specimen was collected for laboratory testing $(54.1 \%)$, and the presence or absence of fever $(50.1 \%)$. Other data elements that tended to be incomplete included the date of rash onset (46.5\%), date of prior measles-rubella-containing vaccination $(44.6 \%)$, and date of investigation (30.8\%).

Table 3: Data elements that were missing among suspected cases of measles and rubella in Ontario, 2009-12

\begin{tabular}{l|c|c}
\hline \multirow{2}{*}{ Data elements } & \multicolumn{2}{|c}{$\begin{array}{c}\text { Suspected cases } \\
\text { (N=497) }\end{array}$} \\
\cline { 2 - 3 } & Number & Proportion (\%) \\
\hline Name & 0 & 0.0 \\
\hline Place of residence & 0 & 0.0 \\
\hline Sex & 4 & 0.8 \\
\hline Age & 3 & 0.6 \\
\hline Date of reporting & 0 & 0.0 \\
\hline Date of investigation & 153 & 30.8 \\
\hline Date of rash onset & 231 & 46.5 \\
\hline Date of specimen collection & 269 & 54.1 \\
\hline Fever & 249 & 50.1 \\
\hline Date of prior measles-rubella \\
vaccine
\end{tabular}

\section{Discussion}

There were no known endemic cases of measles, rubella, or congenital rubella syndrome reported in Ontario between 2009 and 2012. During this period, 27, five and one confirmed cases of measles, rubella, and congenital rubella syndrome respectively were reported. Most imported cases resulted in no or limited onward transmission, supporting the presence of high herd immunity and suggesting effective case investigation and management by health care providers and public health. Further, the estimated effective reproduction number for measles was within the range associated with elimination $(<1)$. Since these diseases are no longer endemic in Ontario, a travelrelated exposure should be indicated for all cases. However, the source of exposure was unknown for three of the 32 cases $(9.4 \%)$ of measles and rubella, suggesting that either there were data quality issues or there was unrecognized disease transmission.

The PAHO definition of an imported case was modified to reflect travel outside Canada rather than the Americas, given that Ontario is one jurisdiction within Canada. As a result, two cases of measles with travel to the United States (U.S.), as well as the ensuing five cases associated with the first case, were classified as imported and import-related respectively. In the first instance, there was documented contact with a measles case from the United Kingdom during travel to the U.S., and therefore this would have met the PAHO definition of import-related. However, on the basis of information recorded in iPHIS the lack of documented measles activity in the area of travel for the second case would have resulted in a classification of unknown under the original PAHO definition.

As Ontario does not have a comprehensive provincial immunization registry, we are not able to assess whether PAHO's criterion for vaccine coverage was met in the population aged one to 40 years. However, according to 2011-12 coverage estimates among school-aged children using data in IRIS, coverage targets were not being 
met for two doses of measles vaccine but were met for a single dose of rubella vaccine. As single antigen measles and rubella vaccines are not available in Ontario, single dose measles vaccine coverage is comparable with rubella coverage (data not shown).

Of the 352 individuals who were investigated as being potentially infected with measles virus between 2009 and 2012, 27 cases were confirmed. For rubella, 145 investigations occurred, and five cases were confirmed. This suggests that the surveillance systems currently in place are sensitive at identifying potential cases and ruling out a diagnosis. However, the annual measles and rubella reporting rate did not meet PAHO's minimum threshold. Ontario may not satisfy this requirement in part because of efficient laboratory testing, which may predispose clinicians to wait for measles and rubella to be ruled out rather than report their suspicions to public health. The suitability of this benchmark in countries targeting elimination was evaluated by Tikhonova et al. (9), who modeled the impact of increasing the investigation rate in the Russian Federation and found a decline in the relative increase in measles cases detected through enhanced versus routine surveillance strategies. The authors cited the need to consider the local epidemiology and associated resource implications. Apart from the national elimination report from the Public Health Agency of Canada (2), we are unaware of studies from other Canadian jurisdictions.

These analyses demonstrate that few suspected cases had all of the required data elements entered in iPHIS, which is a major data quality deficiency. The extent to which immunization information and travel history were missing is a concern, since this information is specified as a key requirement under the Ontario Infectious Diseases Protocol. This suggests that the requisite information is not captured well in iPHIS, which may be due to incomplete data entry or inadequate system infrastructure, wherein iPHIS may not capture the requisite information. It is not known whether there is further information that has not been captured in iPHIS. Despite this, iPHIS remains a centralized data repository for the province, and the active role that the local health units play in conducting surveillance in partnership with the province is a strength of the surveillance system in Ontario.

Lastly, although all specimens that are received by the Public Health Ontario Laboratory for measles and rubella PCR (polymerase chain reaction) testing are forwarded to the National Microbiology Laboratory for genotyping, and results are disclosed to local public health agencies, this information was missing for many cases. This is likely because entry of the information is not mandatory according to current iPHIS data entry guidelines.

Since the transfer of case management responsibilities to Public Health Ontario in 2012, a concerted effort has been made to follow up with local public health units to ensure that suspected measles and rubella cases are appropriately classified as either confirmed or not meeting the definition, and that this is captured accurately in iPHIS. This additional follow-up frequently yields significantly richer information than what is entered in the database. In addition to the development of an investigation form to support local public health units in their investigation of measles and rubella cases, the results from this analysis demonstrate there is a need to improve clarity in iPHIS user guidelines so that critical information such as travel and immunization histories are recorded consistently and comprehensively. Efforts are currently under way within Ontario to address this in order to improve reporting.

\section{Conclusion}

In order for Ontario to satisfactorily demonstrate and maintain elimination of measles, rubella, and congenital rubella syndrome, continued vigilance is required. Although the number of cases identified and the size of outbreaks support sustained elimination, reported immunization coverage and surveillance data quality challenge our ability to document elimination with confidence. 


\section{References}

(1) Final report of the XIX Technical Advisory Group (TAG) Meeting on Vaccine-preventable Diseases of the Pan American Health Organization, held in Buenos Aires, Argentina, July 2011;

http://www.paho.org/hq/index.php?option=com_content\&view=article\&id=1862\&Itemid=2032

(2) Public Health Agency of Canada. Elimination of measles, rubella and congenital rubella syndrome in Canada. Documentation and verification report. December 2011; http://www.phac-aspc.gc.ca/im/vpdmev/measles-rougeole-mrer-eng.php

(3) National Advisory Committee on Immunization. Measles vaccine. In: Canadian limmunization Guide. $7^{\text {th }}$ ed. Ottawa: Public Health Agency of Canada; 2012;

http://www.phac-aspc.gc.ca/publicat/cig-gci/p04-meas-roug-eng.php

(4) National Advisory Committee on Immunization. Rubella vaccine. In: Canadian Immunization Guide. $7^{\text {th }}$ ed. Ottawa: Public Health Agency of Canada; 2012;

http://www.phac-aspc.gc.ca/publicat/cig-gci/p04-rube-eng.php

(5) Ontario Ministry of Health and Long-Term Care. Publicly Funded Immunization Schedules for Ontario August 2011; http://www.health.gov.on.ca/en/public/programs/immunization/docs/schedule.pdf

(6) Pan American Health Organization. Plan of Action for the Documentation and Verification of Measles, Rubella, and Congenital Rubella Syndrome Elimination in the Region of the Americas. Washington, DC. 2011; http://www.paho.org/hq/index.php?option=com_docman\&task=doc_download\&gid=16739

(7) Ministry of Health and Long-Term Care. Infectious Diseases Protocol. Toronto: Queen's Printer for Ontario. 2013;

http://www.health.gov.on.ca/en/pro/programs/publichealth/oph_standards/docs/measles_chapter.p $\mathrm{df}$

(8) De Serres G, Gay NJ, Farrington CP. Epidemiology of transmissible diseases after elimination. Am J Epidemiol 2000; 151:1039-1048; http://aje.oxfordjournals.org/content/151/11/1039

(9) Tikhonova NT, Bichurina MA, Gerasimova AG, Zvirkun OV, Vladimerova NP, Mamaeva T, Lipskaya G, Elsaadany S, Spika JS. Enhanced surveillance for measles in low-incidence territories of the Russian Federation: defining a rate for suspected case investigation. Epidemiol Infect 2011; 139:239-246.

\section{Conflict of interest}

The authors have no conflicts of interest to declare. 